\title{
HACIA UNA EVOLUCIÓN DE LA CONCEPCIÓN DE ANALOGÍA: APLICACIÓN AL ANÁLISIS DE LIBROS DE TEXTO
}

\author{
Fernández González, José1 ; GonZÁlez González, Benigno M. ${ }^{2}$ y Moreno Jiménez, Teodomiro ${ }^{3}$ \\ ${ }^{1}$ Didáctica de las Ciencias Experimentales. Departamento de Didácticas Especiales. Facultad de Educación. Universidad de La \\ Laguna \\ ${ }^{2}$ Formación del Profesorado de La Laguna. Tenerife \\ ${ }^{3}$ IES Anaga. Tenerife \\ jofdez@ull.es \\ bgongonq@gobiernodecanarias.org \\ tmorjim@gobiernodecanarias.org
}

\begin{abstract}
Resumen. Las analogías han contribuido a la construcción y desarrollo del conocimiento científico y a su posterior comunicación, por lo que se consideran fundamentales en el proceso de enseñanza-aprendizaje de las ciencias. En este trabajo se analizan las posiciones acerca de la idea de analogía y se establece una propuesta de su concepción y estructura. Esto ha permitido identificar y estudiar la distribución de las analogías presentes en los libros de texto de enseñanza secundaria, atendiendo a las siguientes variables: fecha de publicación, etapa educativa, materia y curso.
\end{abstract}

Palabras clave. Modelo, analogía, relación analógica.

Summary. Analogies have contributed to the construction and development of scientific knowledge and to its ulterior presentation, because they are essential in the scientific teaching-learning process. In this work the positions about the idea of analogy are analysed and a suggestion about conception and structure analogy is proposed. This fact has permitted the identification and research of the analogy distribution in secondary education textbooks. All this in relation with the following variables: publication date, education level, subjet and school year.

Keywords. Model, analogy, mapping.

\section{INTRODUCCIÓN}

El uso de comparaciones tales como analogías, metáforas, símiles o ejemplos constituye una actividad espontánea de las personas a la hora de dar sentido a lo desconocido. Consecuencia de ello es el papel relevante que han desempeñado las analogías en la construcción de nuevas representaciones científicas. Los científicos las emplean frecuentemente a la hora de elaborar y presentar sus teorías a otros miembros de la comunidad científica (Clement, 1993).

Las analogías también las utiliza el profesorado como recurso didáctico en sus clases, y los autores y editores de libros de texto, ya que constituyen una ayuda para el desarrollo de destrezas de razonamiento científico, para 
la asimilación de conceptos teóricos abstractos e, incluso, para la comprensión de la naturaleza de la ciencia (Lawson, 1993; Wong, 1993; Aragón, 1997).

Las analogías relacionan los contenidos abstractos con la realidad concreta (Dagher, 1994), facilitando su visualización, por lo que constituyen una herramienta que el profesorado puede utilizar -junto con la experimentación y la demostración- para acrecentar la inteligibilidad y plausibilidad de las explicaciones (Osborne y Freyberg, 1985). Facilitan al alumnado ver la ciencia como un «progreso del conocimiento» (Roberts, 1970) y refuerzan su potencial imaginativo, creatividad y habilidad para hacer inferencias y nuevas conexiones mentales. Las analogías permiten construir el nuevo conocimiento y promover, por tanto, el aprendizaje significativo (Bloom, 1992).

\section{DEFINICIONES Y POSICIONES ACERCA DE LA IDEA DE ANALOGÍA}

A pesar del importante papel de las analogías en el proceso enseñanza-aprendizaje de las ciencias, no existe unanimidad entre los investigadores a la hora de definir y concebir la analogía. La bibliografía, por tal motivo, es pródiga en definiciones de analogía, de manera que en una reciente revisión bibliográfica se han encontrado más de una veintena, entre ellas las que se muestran a continuación ${ }^{1}$ :

1) «Siempre que algo es explicado usando comparaciones con otros fenómenos o conceptos estamos haciendo uso de una instrucción con analogías» (Simons, 1984).

2) «La comprensión del tópico por medio del análogo es la base de la analogía» (Donnelly y McDaniel, 1993).

3) «A través de las analogías se puede construir la comprensión de situaciones nuevas por comparación con dominios más familiares del conocimiento» (Wong, 1993).

4) «El objetivo de las analogías es facilitar el desarrollo de modelos conceptuales de nuevas situaciones o conceptos que presentamos a los alumnos por comparación con situaciones familiares» (Iding, 1997).

5) «Una analogía es una comparación entre dos ítems que normalmente no son parecidos» (Davis y Davidson, 1994).

6) «Analogía: relación de semejanza entre cosas distintas» (Piñar, 1998).

7) «Entendemos por analogía aquellos aspectos del discurso explicativo del profesor en los que se usa una situación familiar similar para explicar un fenómeno poco familiar» (Dagher y Cossman, 1992).

8) «Una analogía es un proceso: es el proceso de identificar similitudes entre conceptos diferentes» (Glynn, 1991).

9) «Parecido en algunos detalles entre cosas, que, por otro lado, son diferentes: similitud, correspondencia, paralelismo...» (Webster's Third New International Dictionary, 1986).
10) «Las analogías son cosas o sucesos que son similares a lo que uno está intentando entender» (Glynn, Russell y Noah, 1997).

11) «Una analogía es una correspondencia desde algunos puntos de vista entre conceptos, principios o fórmulas no similares. Más precisamente, es un esquema de relaciones entre características similares de esos conceptos, principios o fórmulas» (Glynn, Britton, Semrud-Clikeman y Muth, 1989; Thiele y Treagust, 1994).

12) «Una analogía es el resultado de identificar similitudes entre dos conceptos. En este proceso hay una transferencia de ideas desde el concepto familiar, llamado análogo, al concepto desconocido, llamado tópico» (Glynn, Law y Doster, 1998).

13) «Las analogías son comparaciones no literales entre dominios de conocimiento a simple vista diferentes» (Zook, 1991; Iding, 1997).

14) «El uso del término analogía se refiere a comparaciones de estructuras entre dos dominios» (Duit, 1991).

15) «Una analogía es una relación entre partes de las estructuras de dos dominios. Por lo tanto, una analogía puede ser vista como un estado de comparación, sobre la base de similitudes, entre las estructuras de dos dominios» (Duit, 1991).

16) «Una analogía se refiere a comparaciones de estructuras entre dominios. Una analogía es una relación entre partes de las estructuras de dos dominios conceptuales y puede ser vista como una comparación fundamentada en la similitud que tienen esas estructuras entre sí» (Treagust, Duit, Joslin y Lindauer, 1992).

17) «Son relaciones que se establecen desde una estructura (análogo) a otra (tópico)» (Newton y Newton, 1995).

18) «La analogía permite la aplicación de estructuras conceptuales previas a nuevos problemas y dominios y, desde aquí, el aprendizaje rápido de nuevos sistemas» (Gentner, Forbus y Kenneth, 1996).

19) «Son las relaciones que se establecen entre la representación de una estructura conceptual (tópico) y un esquema estructuralmente similar del conocimiento base (análogo)» (Halford, 1993).

20) «La analogía es, principalmente, un proceso de comparación en el que se establecen comparaciones de esquemas relevantes entre el análogo y el tópico. Las comparaciones que se establecen de atributos entre el análogo y el tópico son pocas o ninguna» (Gentner, 1983).

En la primera definición se puede observar cómo Simons (1984) fundamenta la analogía en dos términos: comparación y explicación. Asume, por tanto, que las analogías se usan en la explicación de fenómenos o conceptos mediante la comparación. 
«Una analogía se usa con la finalidad de comprender el tópico por medio del análogo». Donnelly y McDaniel (1993) aportan con esta concepción, en su definición de analogía, el término tópico para designar aquello que se intenta comprender y el término análogo para designar el medio del que se va a valer para conseguir dicha comprensión. Aunque no explicitan las características del análogo y del tópico ni cómo tiene lugar dicha comprensión, en esta definición queda claro que la finalidad de la analogía es la comprensión.

Wong (1993) fundamenta la analogía en la comparación de dos situaciones: situación familiar (o dominio familiar) y situación nueva. La finalidad de dicha comparación es la comprensión de la situación nueva.

Iding (1997) establece que el fundamento de la analogía es la comparación entre la situación nueva y la situación familiar, con la finalidad de que los alumnos desarrollen modelos conceptuales de la situación nueva. Esta definición no explicita ni la naturaleza de dichos modelos conceptuales ni la finalidad de los mismos. Sin embargo, se percibe que la finalidad de la analogía es facilitar la comprensión de las situaciones o conceptos nuevos.

Ninguna de las definiciones anteriores hace alusión a la relación entre el análogo y el tópico. Se puede pensar, por lo tanto, que esta relación no existe y que cualquier situación familiar se puede comparar con una situación desconocida para lograr su comprensión. Davis y Davidson (1994) especifican, en este sentido, que se comparan dos ítems -se entiende que se refieren a dos situacionesque normalmente no son parecidos. Aunque esta definición no es muy precisa, introduce un nuevo término que permite relacionar el análogo y el tópico: parecido.

En la analogía se propone una relación de semejanza, pero esta semejanza se da entre cosas distintas. No se apunta, sin embargo, cuántas cosas distintas intervienen en esta relación de semejanza ni tampoco si alguna de ellas debe ser familiar. No se explicita la finalidad de la analogía.

Esta relación de semejanza también se manifiesta en la definición de Dagher y Cossman (1992), en la que queda patente que la finalidad de la analogía es la comprensión de un fenómeno poco familiar. Dicha comprensión se consigue explicando dicho fenómeno a partir de una situación familiar.

Glynn, Law y Dossier (1998) definen la analogía como un proceso en el que se identifican similitudes entre conceptos diferentes. Vuelve a quedar patente, por lo tanto, que la semejanza tiene lugar entre conceptos diferentes. Esta semejanza entre conceptos diferentes queda también de manifiesto en la definición de Webster's Third New International Dictionary (1986). Sin embargo, en estas definiciones no se explica cuántos conceptos diferentes pueden intervenir en dicha relación de semejanza ni si alguno de ellos debe ser familiar. Tampoco se explicita la finalidad de la analogía, es decir, la finalidad del proceso de identificar las similitudes entre los conceptos diferentes. Sin embargo, en una definición posterior, Glynn, Russell y Noah (1997) establecen que las analogías son cosas o sucesos similares que uno está intentando entender. De esta forma dejan claro que en la relación de semejanza interviene un concepto que se quiere entender, pero no clarifican el número y la naturaleza de los otros conceptos. Queda patente que la finalidad de la analogía es la comprensión.

Glynn, Britton, Semrud-Clikeman, Muth (1989) y Thiele y Treagust (1994) coinciden en que una analogía es una comparación desde algunos puntos de vista, de conceptos, principios o fórmulas que son diferentes y que dicha comparación conlleva el establecimiento de un esquema de relaciones entre las características similares de dichos conceptos, principios o fórmulas. Aunque no especifican ni la naturaleza ni el número de conceptos diferentes que intervienen en la comparación, explicitan que entre ellos pueden existir varias características que consiguen relacionarse en base a su semejanza. Esta relación de varias características semejantes da lugar a un esquema de relaciones. Por tanto, cuando estos autores se refieren a esquema de relaciones aluden a que la relación de las características semejantes, entre los conceptos diferentes, puede concebirse mediante un esquema.

El desarrollo de este esquema es la finalidad o el resultado de la analogía, según Glynn, Law y Dossier (1998). Además, estos autores restringen los conceptos que se comparan y los denominan análogo (familiar) y tópico (desconocido). Sin embargo, la aportación más importante de estos autores está en la transferencia de ideas. En la analogía, como resultado del desarrollo del esquema de relaciones entre el análogo y el tópico, tiene lugar una transferencia de ideas -o transferencia de conocimiento- desde el análogo al tópico.

Las definiciones comentadas hasta el momento aportan lo siguiente:

1) La analogía se utiliza en la explicación de una situación o concepto nuevo (tópico) mediante una situación o concepto conocido (análogo).

2) El análogo y el tópico son diferentes, aunque presentan características similares o semejantes.

3) La analogía es un proceso en el que, mediante la comparación del análogo y del tópico, se establece un tejido o trama de relaciones ${ }^{2}$ entre las características similares de ambos. Esta trama de relaciones puede concebirse como un esquema que relaciona las características similares entre análogo y tópico.

4) La finalidad de la analogía es la comprensión de la situación nueva o desconocida, es decir, del tópico. Esta comprensión se consigue porque la analogía facilita el desarrollo de modelos conceptuales del tópico y permite que haya una transferencia de conocimiento desde el análogo al tópico.

Todas las definiciones tratan características similares entre el análogo y el tópico, pero en ninguna figura una clasificación o jerarquización de las mismas. Se podría pensar que en la analogía todas las características que se comparan entre lo desconocido (tópico) y lo familiar (análogo) 
son del mismo tipo. O que todas valen: sólo es necesario que sean características semejantes. Esto último equivale a suponer que la transferencia de conocimiento desde el análogo al tópico se fundamenta, por igual, en todas las características semejantes entre ambos.

Las definiciones que se van a explicar a continuación ponen de manifiesto que esto no es así, que existen diferentes tipos de características semejantes -o diferentes tipos de semejanza- entre el análogo y el tópico y que no todas tienen igual ponderación en la transferencia de conocimiento entre ambos.

Zook e Iding (1997) advierten de ello cuando introducen en su definición de analogía la expresión comparaciones no literales. Las analogías son, por tanto, comparaciones con peculiaridades. Esta definición vislumbra la existencia de diferentes tipos de comparaciones en la analogía. Es una definición de analogía que incita a la reflexión, aunque es poco precisa y clarificadora.

Las definiciones de Duit (1991) son, en este sentido, más clarificadoras. Restringen las relaciones a comparaciones de estructuras, es decir, a comparaciones entre características que constituyen el fundamento conceptual del análogo y del tópico. En la analogía se comparan, por tanto, características estructurales. Además, Duit precisa que sólo se comparan las características estructurales que son semejantes.

Cabe señalar la observación hecha por Duit, aunque no forme parte de la definición de analogía aportada por este investigador, al señalar que en la comparación de las características estructurales semejantes se desarrollan tanto el análogo como el tópico. Es decir, siempre que se usa una analogía no sólo se explica el tópico sino que también se desarrolla el análogo (Duit, 1991).

Treagust, Duit, Joslin y Lindauer (1992) también definen la analogía en los términos anteriores.

Del mismo modo Newton y Newton (1995) definen la analogía como relaciones -comparaciones- entre estructuras: la del análogo y la del tópico. Asignan a estas comparaciones un sentido, el que va desde el análogo al tópico. Dicho sentido en el que se establece la comparación lleva implícito la transferencia de conocimiento desde el análogo al tópico. Sin embargo, en esta definición no se advierte que las estructuras que se comparan deban ser similares. Tampoco se especifica si dichas estructuras son las estructuras totales del análogo y del tópico o sólo una parte de las mismas.

Gentner, Forbus y Kenneth (1996) definen la analogía como aquello que permite aplicar -transferir- el conocimiento de estructuras conceptuales previas -estructura del análogo- a nuevos problemas y dominios -estructura del tópico- y, desde aquí, el aprendizaje del tópico. Si bien esta definición explica que la transferencia de conocimiento desde el análogo al tópico es estructural, no explicita el tipo de semejanza estructural del análogo y del tópico, ni si dichas estructuras son las estructuras totales o sólo una parte de las mismas.
Halford sí explicita, en su definición de analogía, la similitud entre las estructuras del análogo y del tópico que se comparan o relacionan. Indica, además, que dichas estructuras se pueden representar mediante esquemas. No explicita tampoco si esa similitud afecta a la totalidad o a una parte de las estructuras.

Se puede afirmar, en base a las últimas definiciones, lo siguiente:

1) Entre el análogo y el tópico existe un tipo de semejanza, la semejanza estructural.

2) En el proceso de comparación que tiene lugar en la analogía entre el análogo y el tópico interviene la semejanza estructural.

3) La semejanza estructural afecta sólo a parte de las estructuras del análogo y del tópico.

4) En la analogía tiene lugar una transferencia de conocimiento desde parte de la estructura del análogo a parte de la del tópico. Es decir, la transferencia de conocimiento se produce desde la semejanza estructural.

5) Las estructuras del análogo y del tópico se pueden representar mediante esquemas.

Estas afirmaciones reflejan la gran importancia que tiene en la analogía la semejanza estructural. Sin embargo, son poco explícitas en lo referente a cómo representar la estructura conceptual del análogo o del tópico. Tampoco ponen de manifiesto la existencia de otro tipo de semejanza en la analogía, diferente de la semejanza estructural.

Gentner (1983), en el denominado modelo estructural de razonamiento analógico, admite que en el proceso de comparación existe una transferencia de conocimiento desde el análogo al tópico, y que sólo se transfiere aquello que es semejante y de interés del análogo, hecho que caracteriza un tipo de razonamiento imprescindible cuando se usa la analogía: el «razonamiento analógico». Con estas argumentaciones propone la definición de analogía (que figura en el número 20 de las encontradas en la revisión bibliográfica):

«La analogía es, principalmente, un proceso de comparación en el que se establecen comparaciones de esquemas relevantes entre el análogo y el tópico. Las comparaciones que se establecen de atributos entre el análogo y el tópico son pocas o ninguna» (Gentner, 1983).

El modelo estructural, del que forma parte esta definición, admite que tanto el conocimiento del análogo como el del tópico pueden representarse de forma que se reconozca lo siguiente:

1) Que el análogo y el tópico están constituidos por elementos («componentes» en lo sucesivo, según nuestra terminología) y que éstos forman parte de su estructura.

2) Que cada elemento («componente») viene caracterizado por una serie de propiedades o características denominadas atributos. 
3) Que entre los elementos («componentes») existen conexiones (que identificamos como «nexos»), que son las correlaciones o comparaciones entre estos elementos («componentes»).

4) Que estas conexiones («nexos») constituyen la estructura del análogo y del tópico.

Con ella se acepta que la información importante que se transfiere entre el análogo y el tópico está contenida en los nexos y no en los atributos y, por tanto, las comparaciones relevantes entre análogo y tópico son las de los nexos, más que las de los componentes o atributos. Queda pues establecido que:

- la analogía es un proceso de comparación de nexos semejantes, independientemente de los componentes de los que esos nexos estén formando parte (Gentner, 1989);

- las comparaciones importantes son entre nexos semejantes.

Este modelo estructural del razonamiento analógico distingue las comparaciones de atributos de componentes -a las que denomina comparaciones de orden inferiorde las comparaciones de nexos -a las que denomina de orden superior. Establece que la finalidad de la analogía es la comprensión del tópico por transferencia de conocimiento desde el análogo al tópico.

En otras palabras, en el modelo estructural, la transferencia de conocimiento se produce por comparación de nexos semejantes (semejanza estructural). A esto último se le da el nombre de principio de sistematicidad.

La definición de Gentner (1983) lleva implícito el principio de sistematicidad al establecer que en la analogía se comparan nexos relevantes. En la analogía, los nexos semejantes en el análogo y el tópico van a ser, por lo tanto, los nexos relevantes. Además, en la transferencia de conocimiento no interviene toda la estructura del análogo y del tópico. Este hecho se denomina, en el modelo estructural, con el nombre de restricción estructural en la analogía y aparece reflejado en la figura 1.

Figura 1

Nexos relevantes.

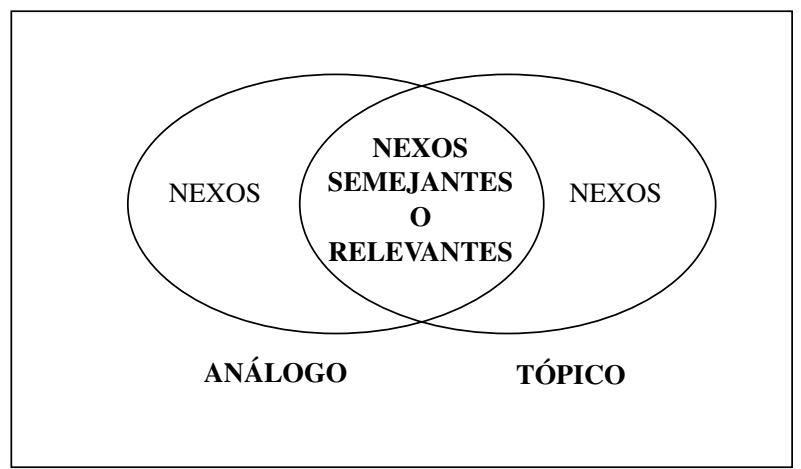

\section{CONCEPCIÓN DE ANALOGÍA}

La comparación relaciona una situación familiar al alumno, denominada análogo, con el concepto teórico y abstracto desconocido, denominado tópico. Facilita, por tanto, la conexión entre el conocimiento adquirido previamente y lo que se pretende aprender para que tenga lugar una transferencia de conocimiento desde el análogo al tópico.

El proceso mental, estructural y funcional que comprende la comparación y la transferencia de conocimiento desde el análogo al tópico se denomina analogía. El razonamiento que tiene lugar cuando se usa la analogía y que permite que sólo se transfiera aquello que es semejante y útil del análogo para la comprensión del tópico se denomina razonamiento analógico.

El alumno dispone, de esta forma, mediante la analogía y el razonamiento analógico asociado a ella, de un modelo mental adecuado e imprescindible para posteriores aprendizajes, constituido por la estructura común de las relaciones que se establecen entre el análogo y el tópico, tal como se refleja en la figura 2.

Figura 2

Estructura de la analogía, base del razonamiento analógico.

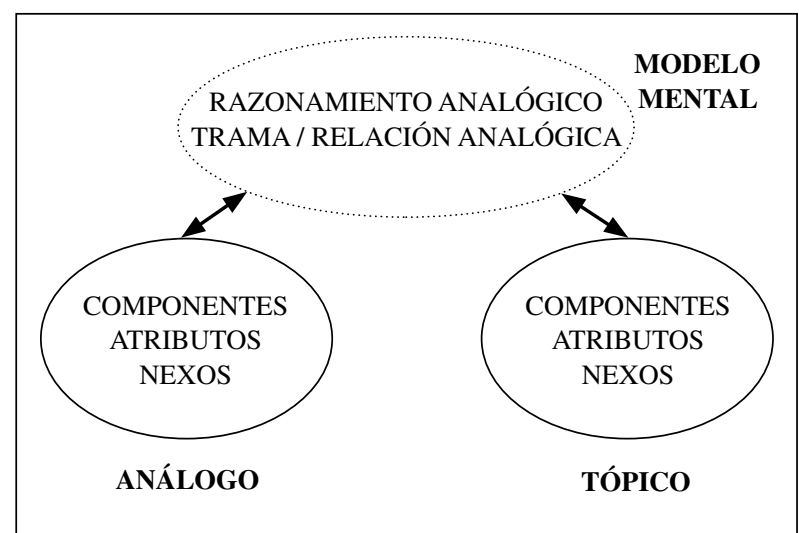

Siempre que se usa una analogía no sólo se desarrolla el tópico sino también el análogo, debido a que el análogo puede verse desde una nueva perspectiva, la perspectiva del tópico. Por este motivo, el profesor puede alternar en sus explicaciones el rol del análogo y del tópico y contribuir a un aprendizaje más profundo del tópico. Esta es la razón por la que en la figura 2 , donde se ha representado la estructura de una analogía, se ha relacionado el análogo, el tópico y el modelo mental con flechas dobles.

El análogo y el tópico son diferentes, aunque presentan características similares o semejantes. La analogía puede concebirse como un proceso en el que, mediante la comparación del análogo y del tópico, se establece una trama de relaciones -relación analógica- entre las características similares de ambos. Se puede imaginar esta 
trama de relaciones como un entretejido que relaciona las características similares del análogo y del tópico.

Pero, ¿qué tipo de características similares forman parte de la trama de relaciones? Para contestar a esta pregunta se admite, antes, lo siguiente (Fig. 2, Cuadro I):

1) Que tanto el análogo como el tópico están formados en su estructura por componentes.

2) Que cada componente se caracteriza por una serie de propiedades, características o atributos.

3) Que entre los componentes existen nexos que son las correlaciones o comparaciones entre estos componentes.

4) Que estos nexos constituyen la estructura del análogo y del tópico.

5) Las comparaciones de nexos (características estructurales) semejantes del análogo y del tópico son la parte fundamental de la trama o relación analógica. Estas comparaciones pueden ser una semejanza estructural si afecta a su configuración o pueden alcanzar el significado y la función: semejanza semántica y funcional.

6) Las comparaciones de atributos (características superficiales) semejantes entre el análogo y el tópico tienen un carácter más secundario en la relación analógica. Se suelen denominar semejanza superficial.

Cuadro I

Esquema acerca de nexos y atributos.

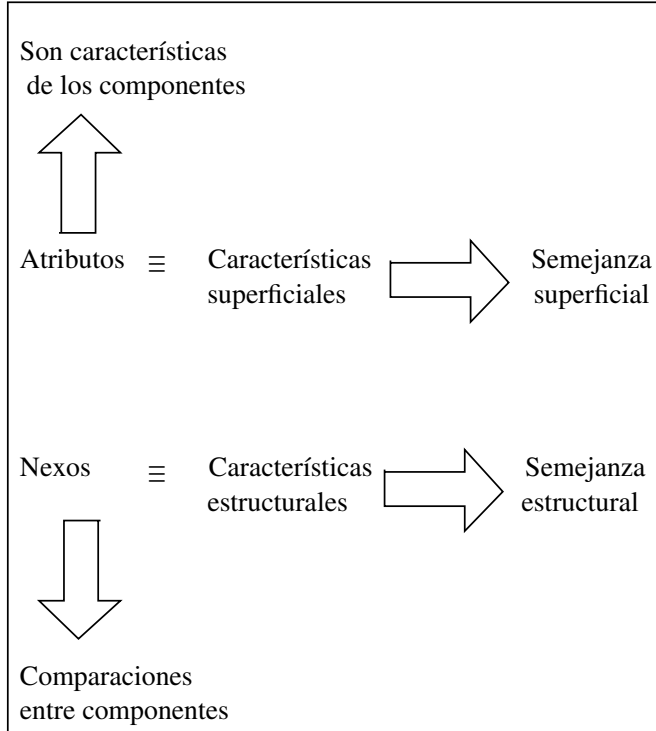

Con el convencimiento de que no se puede dar una definición de analogía por cuanto representa todo un proceso complejo, sí que se intenta dar una respuesta simplificada basada en las argumentaciones anteriores:
«Una analogía es una propuesta representativa de las estructuras del análogo y del tópcio. Mediante una trama de relaciones se comparan, fundamentalmente, los nexos semejantes entre ambos.

»Su finalidad es la comprensión y el aprendizaje del tópico mediante la transferencia de conocimiento del análogo al tópico.

»Las comparaciones de atributos semejantes tienen un carácter secundario» (González, 2002).

\section{COMPARACIÓN CON OTROS AUTORES}

A continuación se hace un análisis comparativo entre la concepción de analogía argumentada en el apartado anterior y las distintas definiciones de analogía encontradas en la revisión bibliográfica. El análisis comienza con las cinco primeras definiciones que se explicitaron anteriormente.

En estas definiciones queda patente que la analogía se fundamenta en la comparación y en la explicación del tópico para que se produzca su comprensión y aprendizaje. También queda patente que se compara una situación familiar, el análogo, con una desconocida o nueva, el tópico. En esta comparación, los alumnos desarrollan modelos mentales del tópico, modelos que están directamente relacionados con la trama de relaciones entre el análogo y el tópico.

Todos estos aspectos se tienen en cuenta en el planteamiento de analogía que se ha propuesto.

Otro grupo de definiciones de analogía, como las que comprenden de la 6 a la 12 inclusive, se analizan a continuación.

En este grupo de definiciones queda patente que análogo y tópico son situaciones o conceptos diferentes entre los que existe una relación de semejanza y que ésta se puede visualizar mediante un esquema -o trama de relaciones- que compare las características similares de ambos. También se pone de manifiesto que en esta comparación existe una transferencia de ideas.

Estos aspectos están también presentes en el planteamiento de analogía propuesto.

Las definiciones 13 a la 19 inclusive contemplan la existencia de un tipo de semejanza entre el análogo y el tópico, la semejanza estructural.

Confirman que es la semejanza estructural la única que interviene en el proceso de comparación entre el análogo y el tópico para que tenga lugar la transferencia de conocimiento, y que ésta no afecta a la totalidad de la estructura de ambos. Además, revelan que las estructuras del análogo y del tópico, al igual que la trama de relaciones entre ambos, se pueden representar por medio de esquemas. 
La última definición del análisis es la de Gentner (1983), definición en la que se constata la existencia de otro tipo de semejanza, de menor importancia, denominada semejanza superficial o semejanza entre atributos.

Esta definición forma parte del denominado modelo estructural de razonamiento analógico, modelo que admite que tanto el análogo como el tópico están constituidos por componentes, atributos de componentes y nexos entre componentes y que son los nexos los que conforman su estructura. Implica que la semejanza estructural es una semejanza entre nexos.

Como se puede observar, todos estos aspectos se recogen en el planteamiento de analogía.

Sin embargo, ninguna de las definiciones anteriores es capaz de concretar el tipo de semejanza que puede existir entre los nexos. Esta puntualización se debe a Holyoak y Thagard (1989) y Thagard (1992) al afirmar que entre los nexos puede existir semejanza estructural y semejanza semántica.

La semejanza estructural incluye aquellos nexos que presentan componentes con configuraciones similares. Se trata de nexos que presentan relaciones o proporciones semejantes entre sus componentes. La semejanza semántica es la que presentan los nexos que tienen significados semejantes, es decir, que tienen un comportamiento o funcionamiento semejante.

La aportación de Holyoak y Thagard es muy interesante y coherente con la propuesta que se ha planteado de analogía. Basta con reflexionar en la mención que en ella se hace de «nexos semejantes entre ambos». Al mencionar la semejanza entre nexos se alude de manera implícita a los dos tipos de semejanza que proponen los investigadores: la semejanza estructural y la semejanza semántica.

\section{INTERPRETACIÓN DE LA CONCEPCIÓN DE ANALOGÍA}

La analogía del sistema solar como representación del átomo de Rutherford va a servir de ejemplo para interpretar la propuesta de concepción de analogía planteada $\mathrm{y}$ argumentada anteriormente.

Se trata de reconocer en esta analogía el tópico, el análogo, los componentes del tópico y del análogo, los atributos y los nexos de ambos. Asimismo se resaltará la comparación de nexos semejantes o relevantes, la semejanza estructural, semejanza funcional o semántica, la semejanza superficial y la escenificación de la trama o relación analógica.

En esta analogía, el análogo (lo familiar y conocido) es el sistema solar y los componentes son el Sol y los planetas. El tópico (lo que se propone enseñar y desconocido) es el átomo de Rutherford y sus componentes son el núcleo y los electrones.
Los atributos del sistema solar son, por ejemplo, el tamaño del Sol y los planetas, su forma, su temperatura, su color, etc. Los atributos del átomo de Rutherford son, por ejemplo, la forma y tamaño -geometría y disposición-del núcleo y de los electrones.

Los nexos que existen entre los componentes del sistema solar son los siguientes: distancia entre el Sol y los planetas, fuerza atractiva entre el Sol y los planetas, giro de los planetas alrededor del Sol, mayor masa del Sol que la de los planetas y mayor temperatura del Sol que la de los planetas. Los nexos que existen entre los componentes del átomo de Rutherford son los siguientes: distancia entre el núcleo y los electrones, fuerza atractiva entre el núcleo y los electrones, giro de los electrones alrededor del núcleo y mayor masa del núcleo que la de los electrones.

\section{NEXOS DEL ANÁLOGO}

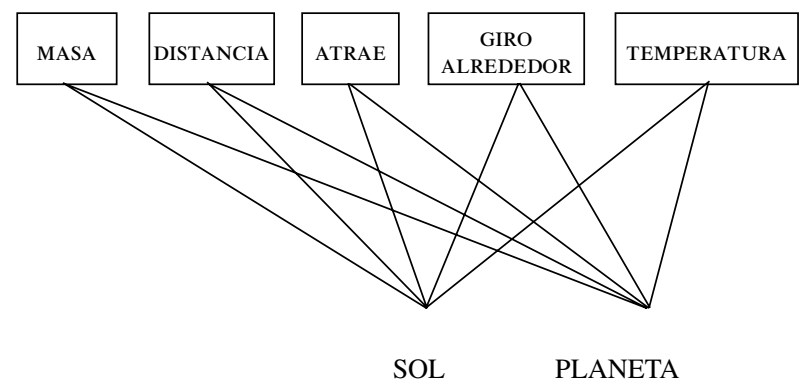

NEXOS DEL TÓPICO

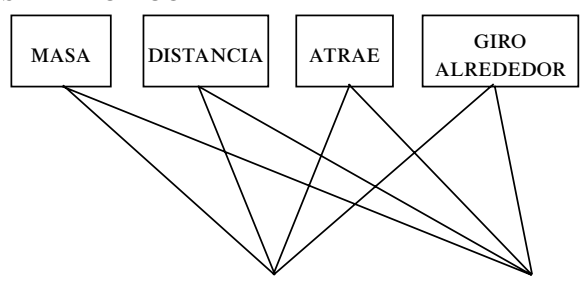

NÚCLEO ELECTRÓN

La transferencia de conocimiento desde el análogo al tópico se produce, fundamentalmente, como resultado de comparaciones entre nexos semejantes -comparaciones de orden superior, como los que se muestran en el siguiente esquema:
Mayor masa del Sol que de los planetas Mayor masa del núcleo que
Distancia del Sol a los planetas de los electrones
Fuerza atractiva entre el Sol y los planetas Distancia del núcleo a los electrones
Giro de los planetas alrededor del Sol
Fuerza atractiva entre el núcleo y los electrones

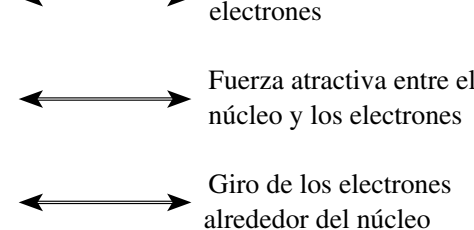


Así pues, la transferencia de conocimiento tiene lugar entre nexos semejantes. Por este motivo, cuanto mayor sea el grado de semejanza en una analogía, mayor va a ser la cantidad de conocimiento relevante que pueda transferirse desde el análogo hacia el tópico.

La figura 3 muestra la estructura de la analogía del sistema solar:

Figura 3

Estructura de una analogía.

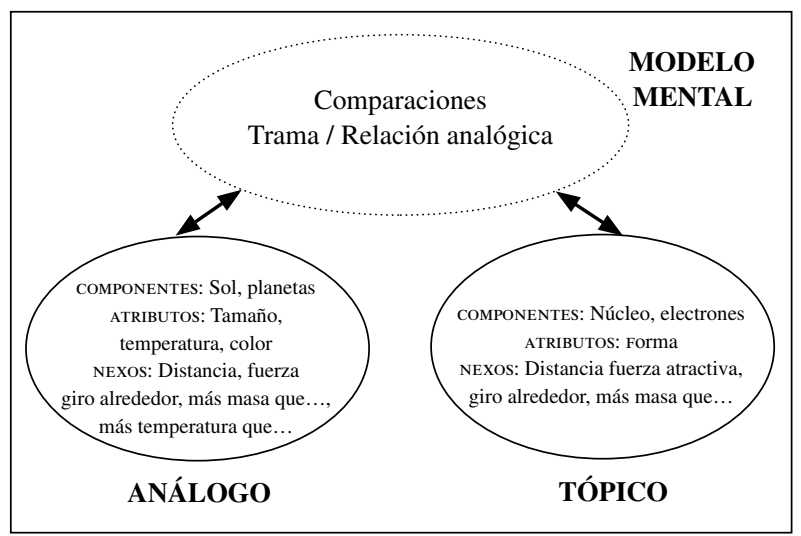

En la analogía del sistema solar existe una gran semejanza entre el análogo y el tópico porque la mayoría de los nexos presentes en ambos son semejantes. En otras palabras, el subconjunto de los nexos relevantes es bastante extenso, ya que incluye la mayoría de los nexos del análogo y del tópico.

Estos nexos son semejantes en su configuración (semejanza estructural):

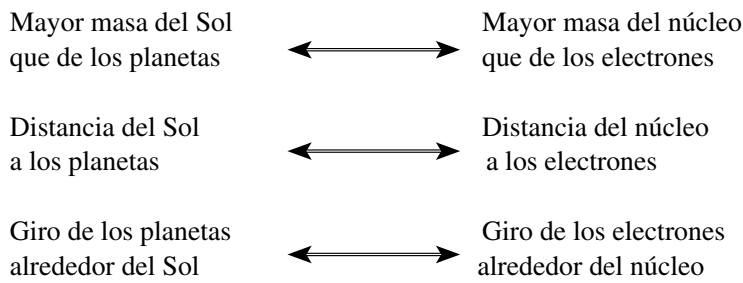

O son semejantes en su significado en relación con la función que desempeñan (semejanza semántica y funcional), de manera que son los responsables de la semejanza en el comportamiento o funcionamiento del análogo y del tópico. Fijémonos en el último de los nexos anteriores y en el siguiente:
De hecho, tanto en el sistema solar como en el átomo de Rutherford, la fuerza atractiva determina el giro a una cierta distancia entre cuerpos de diferente masa.

Siempre existirán nexos que no se corresponden, que no tienen un semejante y, por lo tanto, que no serán relevantes para la analogía, como es el caso del nexo «más temperatura que». Este nexo es descartado en este proceso de comparaciones del sistema solar y el átomo de Rutherford.

En definitiva, en esta analogía la semejanza estructural y semántica puede dirigir a los alumnos hacia el descubrimiento de los siguientes nexos relevantes: «distancia» (Sol, planeta y núcleo, electrón), «fuerza atractiva» (Sol, planeta y núcleo, electrón), «gira alrededor de» (planeta, Sol y electrón, núcleo) y «más masa que» (Sol, planeta y núcleo, electrón).

La semejanza superficial, es decir, las comparaciones entre los atributos del análogo y del tópico, puede dar lugar a que se transfieran atributos erróneos para el aprendizaje. Este hecho es especialmente importante en los alumnos más jóvenes, porque son los alumnos que dan mayor importancia a esta semejanza, como consecuencia de tener más dificultades para entender la analogía (Gentner y Gentner, 1983).

Un ejemplo de ello se tiene en el color amarillo del Sol, debido a su elevada temperatura. Este atributo puede dirigir a los alumnos a pensar que tiene su semejante en el núcleo y, por este motivo, a transferir el nexo «más caliente que».

\section{ANÁLISIS DE LA DISTRIBUCIÓN DE ANALOGÍAS EN LOS LIBROS DE TEXTO}

Se ha llevado a cabo un análisis de las analogías en los libros de texto que contempla las siete editoriales de educación secundaria que mayor arraigo tienen dentro del ámbito nacional (González y Moreno, 1998; González, Moreno y Fernández, 2000; González, 2002). Son las siguientes: Ecir, Edebé, Anaya, McGraw Hill, SM, Oxford educación (u Oxford) y Santillana. Conforman una muestra de 84 libros de texto que tratan sobre las siguientes materias de educación secundaria: ciencias de la naturaleza, física y química, biología y geología, física, química, biología, geología y ciencias de la tierra y medio ambiente.

El número de textos de cada editorial que forman parte de este análisis no es el mismo, puesto que, en el momento de realizar el estudio, algunas editoriales no habían publicado el texto correspondiente a determinadas materias. Aunque es habitual que cada editorial publique un único ejemplar por curso y materia, la editorial SM publica dos ejemplares en cada uno de los cuatro cursos de la educación secundaria obligatoria. Así sucede en ciencias de la naturaleza de $1^{\circ}$ y $2^{\circ}$ de ESO (Explora y Ozono), en biología y geología de $3^{\circ}$ y $4^{\circ}$ de ESO (Biosfera y Darwin) y en física y química 
de $3^{\circ}$ y $4^{\circ}$ de ESO (Interacción y Newton). La finalidad, según los autores y editores de SM, es ofrecer al profesor y a los alumnos dos líneas didácticas diferentes: a) por un lado, una línea más innovadora, en la que se exploran las ideas previas de los alumnos y se fomenta la discusión y participación de éstos en clase a través de numerosas actividades prácticas. Pertenecen a esta línea los textos que presentan la denominación Explora, Biosfera e Interacción. En esta investigación se denomina a esta línea $S M(I) ; b)$ por otro lado, se presentan una línea más habitual, con mayor profundidad conceptual y actividades numéricas. Pertenecen a ella los textos que muestran la denominación Ozono, Darwin y Newton. Se denomina línea $S M(T)$.

El análisis de los 84 libros de texto ha permitido identificar 399 analogías, de acuerdo con la concepción de analogía que se ha argumentado. A continuación se muestran algunos de los resultados del mismo (González, 2002), concretamente aquéllos que están relacionados con la distribución de las analogías en los libros de texto.

\section{Distribución de las analogías en los textos de cada una de las editoriales}

El número total de analogías identificadas (399) en relación con el número de libros de texto analizados (84) representa la cantidad promedio ${ }^{3}$ de analogías por cada libro de texto. Se obtiene una cifra de 4,75 analogías por cada libro de texto. Así, por ejemplo, en el caso de los textos de química, el número total de analogías identificadas es 28 y el de textos analizados 6 , datos que reportan un promedio de 4,67 analogías por cada libro de texto. Estos promedios son bajos si se comparan con el de otras investigaciones llevadas a cabo con libros de texto de otros países.

Así, Curtis y Reigeluth (1984) obtuvieron un promedio de 8,3 analogías por cada libro de texto cuando analizaron una muestra de 26 libros de texto americanos de ciencias -biología, ciencias naturales, física, química, ciencias de la tierra y geología- de secundaria. Thiele y Treagust (1994), en un análisis de 10 libros de texto de química de secundaria australianos, obtuvieron un promedio de 9,3 analogías por cada libro de texto.

Tabla I

Número de analogías por materias en ESO y en bachillerato.

\begin{tabular}{|c|c|c|c|c|c|c|c|c|c|c|c|c|c|}
\hline Nivel educativo & \multicolumn{4}{|c|}{ ESO } & \multicolumn{6}{|c|}{ BACHILLERATO } & \multirow{2}{*}{ TOTAL } \\
\hline Materias & CCNN & FYQ & BYG & TOTAL & FYQ & BYG & F & Q & B & G & CTMA & TOTAL & \\
\hline Núm. de textos & 16 & 16 & 16 & 48 & 7 & 7 & 7 & 6 & 7 & 1 & 1 & 36 & 84 \\
\hline Núm. de analogías & 85 & 70 & 56 & 211 & 43 & 45 & 43 & 28 & 28 & 0 & 1 & 188 & 399 \\
\hline \% de analogías & 21,3 & 17,5 & 14,0 & 52,8 & 10,8 & 11,3 & 10,8 & 7,02 & 7,02 & 0 & 0,251 & 47,2 & 100 \\
\hline
\end{tabular}

Gráfico 1

Promedio de analogías por cada texto.

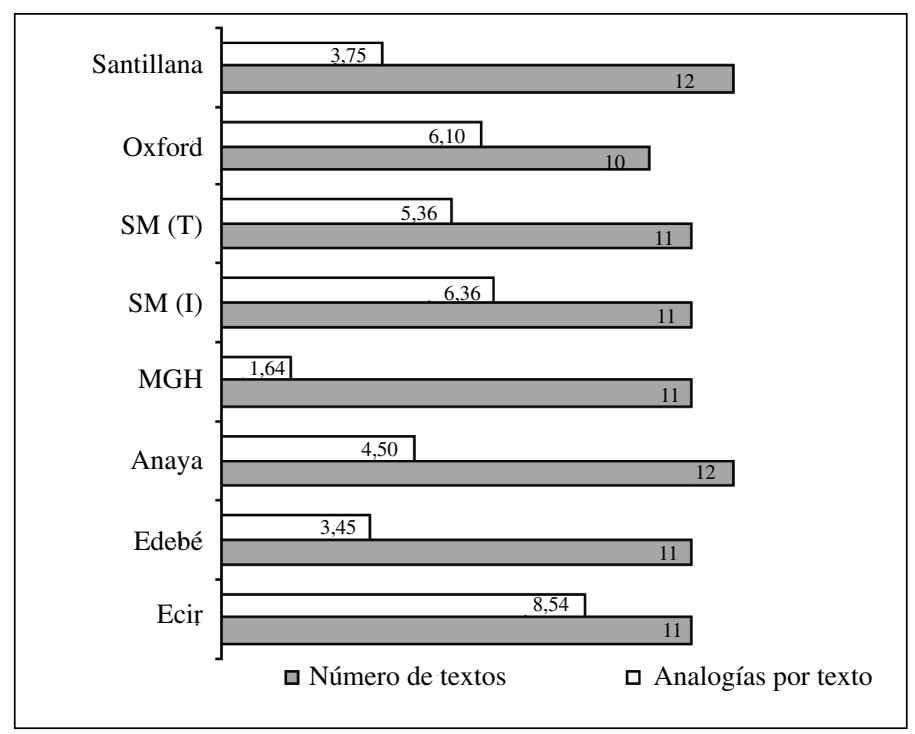


Este promedio es diferente de una editorial a otra, tal como se refleja en el gráfico 1. En éste aparecen dos columnas, una más larga, que indica el número de textos en cada editorial, y otra más corta, que señala el porcentaje de promedio de analogías por libro de texto.

Así, en los 11 libros de texto de la editorial Ecir analizados, se han identificado 94 analogías. Si se divide el número de analogías identificadas (94) por el número de libros de texto de esta editorial (11) se obtiene un promedio de 8,54 analogías por cada libro de texto. Representa el promedio más alto de la muestra de editoriales y el que más se aproxima al de las investigaciones llevadas a cabo con editoriales de otros países.

En los 11 libros de texto de la editorial MGH analizados se han identificado 18 analogías. El número de analogías (18) entre el número de libros de texto de esta editorial (11) resalta un promedio de 1,64 analogías por cada libro de texto. Representa el promedio más bajo que ostenta una editorial de las que conforman la muestra y el que más se aleja de las investigaciones llevadas a cabo con editoriales de otros países.

\section{Analogías en los textos según el año de publicación}

También difiere de una a otra editorial el período en que se publicaron los diferentes libros de texto. La editorial que presenta libros de texto más recientes es la editorial Oxford, con fechas de publicación que oscilan entre los años 1998 y 2000, y es además la que presenta uno de los promedios más altos: 6,1 analogías por cada libro de texto.

Otra editorial con un promedio alto que también tiene publicaciones comprendidas entre los años 1997 y 1999 es la editorial SM. En cada una de sus dos líneas editoriales, señaladas como $\mathrm{SM}(\mathrm{I})$ y $\mathrm{SM}(\mathrm{T})$, presenta 6,36 y 5,36 analogías por texto.

Si se tiene en cuenta que una de las editoriales que presenta libros de texto más antiguos es la editorial McGraw Hill y que justo es esta editorial la que presenta el promedio más bajo, parece lógico suponer que existe una cierta relación entre la fecha de publicación de los diferentes libros de texto y la cantidad de analogías que éstos presentan. Es decir, se podría pensar que los textos más recientes han ido incorporando un repertorio mayor de analogías en sus explicaciones.

Para confirmar esta hipótesis se debe conocer el número de analogías que presentan los textos analizados y su fecha de publicación. Se pueden contabilizar las analogías existentes en los textos y el año en que fueron publicados en un intervalo comprendido entre los años 1995 y 2000, ambos inclusive, y dividir el número de analogías encontradas en los textos publicados en un año determinado entre el número de textos publicados en dicho año. El gráfico 2 refleja estos resultados:

Aunque se aprecia una tendencia a incorporar un mayor número de analogías en los textos de publicación más reciente, no se puede afirmar que ésta sea la única variable que justifique el elevado margen que existe entre unas editoriales y otras. Es decir, no se puede afirmar que los textos de más reciente publicación incorporen un repertorio mayor de analogías en sus explicaciones.

El año 1999 corrobora la afirmación anterior por los bajos resultados. Además, los datos referentes al año 2000 no son representativos, puesto que corresponden a una única editorial, la editorial Oxford, debido a la disponibilidad de la muestra.

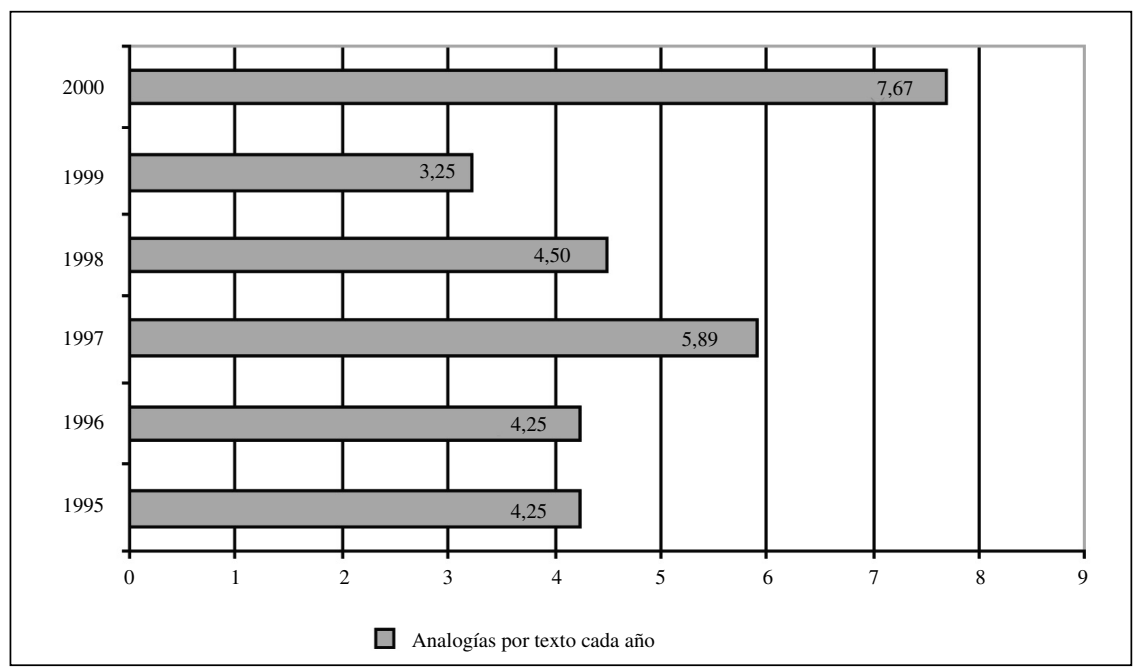


A la hora de confeccionar los libros de texto, probablemente son las preferencias de los autores y editores las variables que más influyen en la determinación de cada editorial a incorporar analogías en sus textos. Estas preferencias pueden estar condicionadas por el conocimiento y la consideración que autores y editores tengan sobre las analogías y por aspectos económicos. No cabe duda de que extenderse en la adecuación de las unidades didácticas supone elaborar un texto más amplio y, por consiguiente, más caro.

\section{Distribución de las analogías por etapas educativas (ESO y bachillerato)}

La tabla I refleja cómo se distribuyen las analogías que se han identificado en los textos de ESO y de bachillerato. Refleja que, de las 399 analogías identificadas, 211 analogías (el 52,9\%) se encuentran localizadas en textos de ESO y 188 (el 47,1\%) en textos de bachillerato. Se puede admitir, por lo tanto, que autores y editores de libros de texto hacen un mayor esfuerzo para facilitar la comprensión de los conceptos a los alumnos de los niveles más bajos.

Sin embargo, los porcentajes de la distribución de analogías en ESO y bachillerato son muy variables cuando se concretan en cada editorial, tal como puede apreciarse en la tabla II.

Mientras la editorial Ecir destina el 60,6\% de sus analogías a los textos de ESO y MGH el 66,7\%, la editorial SM (T) es la que más se desvía de esta posición al destinar a los textos de ESO sólo el 32,2\%.

\section{Distribución de las analogías en cada una de las materias}

El análisis de los diferentes libros de texto ha revelado que el rango de inclusión de las analogías varía mucho de unos a otros. La tabla III muestra la distribución de las analogías por materias, tanto de ESO como de bachillerato, de manera que los porcentajes están referidos al número total de analogías identificadas (399 analogías).

Tabla II

Porcentajes correspondientes a la distribución de analogías en ESO y bachillerato.

\begin{tabular}{|c|c|c|c|c|c|}
\hline EDITORIAL & $\begin{array}{l}\text { ANALOGÍAS } \\
\text { ESO }\end{array}$ & $\begin{array}{l}\text { ANALOGÍAS } \\
\text { ESO }(\%)\end{array}$ & $\begin{array}{c}\text { ANALOGÍAS } \\
\text { BACHILLERATO }\end{array}$ & $\begin{array}{c}\text { ANALOGÍAS } \\
\text { BACHILLERATO }(\%)\end{array}$ & $\begin{array}{l}\text { TOTAL } \\
(\%)\end{array}$ \\
\hline Ecir & 57 & 60,6 & 37 & 39,4 & 100 \\
\hline Edebé & 20 & 52,6 & 18 & 47,4 & 100 \\
\hline Anaya & 20 & 37 & 34 & 63 & 100 \\
\hline MGH & 12 & 66,7 & 6 & 33,3 & 100 \\
\hline $\begin{array}{c}\text { SM(I) } \\
\text { SM(BACH) } \\
\text { SM(T) }\end{array}$ & $\begin{array}{l}30 \\
19\end{array}$ & $\begin{array}{l}42,9 \\
32,2\end{array}$ & 40 & $\begin{array}{l}57,1 \\
67,8\end{array}$ & $\begin{array}{l}100 \\
100\end{array}$ \\
\hline Oxford & 32 & 52,5 & 29 & 47,5 & 100 \\
\hline Santillana & 21 & 46,7 & 24 & 53,3 & 100 \\
\hline
\end{tabular}

Tabla III

Distribución de las analogías por materias en ESO y bachillerato.

\begin{tabular}{|c|c|c|c|c|c|c|c|c|c|c|c|}
\hline $\begin{array}{c}\text { MATERIAS } \\
\text { CURSOS }\end{array}$ & $\begin{array}{c}\text { CCNN } \\
1^{\circ} \text { y } 2^{\circ} \\
\text { ESO }\end{array}$ & $\begin{array}{c}\text { FyQ } \\
3^{\circ} \text { y } 4^{\circ} \\
\text { ESO }\end{array}$ & $\begin{array}{c}\text { FyQ } \\
\mathbf{1}^{\circ} \\
\text { BACH }\end{array}$ & $\begin{array}{c}\text { ByG } \\
3^{\circ} \text { y }^{\circ} \\
\text { ESO }\end{array}$ & 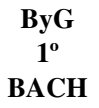 & $\begin{array}{c}\mathbf{F} \\
2^{\mathbf{o}} \\
\mathbf{B A C H}\end{array}$ & $\begin{array}{c}\mathbf{Q} \\
\mathbf{2}^{\mathbf{o}} \\
\mathbf{B A C H}\end{array}$ & $\begin{array}{c}\text { B } \\
2^{\circ} \\
\text { BACH }\end{array}$ & $\begin{array}{c}\mathbf{G} \\
2^{\circ} \\
\text { BACH }\end{array}$ & $\begin{array}{c}\text { СТМА } \\
2^{\circ} \\
\text { ВАСН }\end{array}$ & TOTAL \\
\hline $\begin{array}{l}\text { Núm. de } \\
\text { analogías }\end{array}$ & 85 & 70 & 43 & 56 & 45 & 43 & 28 & 28 & 0 & 1 & 399 \\
\hline $\begin{array}{c}\text { Analogías } \\
\%\end{array}$ & 21,3 & 17,5 & 10,8 & 14,0 & 11,3 & 10,8 & 7,02 & 7,02 & 0 & 0,251 & 100 \\
\hline
\end{tabular}


El mayor porcentaje de analogías $(21,3 \%)$ se da en los libros de texto de ciencias de la naturaleza de $1^{\circ}$ y $2^{\circ}$ de ESO. Incluso en el caso de considerar, en el cálculo de los porcentajes, una única línea de edición (editorial $\mathrm{SM}$ ), el porcentaje continuaría siendo el más alto para los textos de ciencias naturales de la ESO. Este hecho pone de manifiesto que los autores y editores de libros de texto de ciencias se inclinan por utilizar un mayor número de analogías en el nivel más bajo de la enseñanza secundaria obligatoria, el que corresponde al primer ciclo.

La distribución en cada uno de los dos cursos del primer ciclo de la ESO es similar, tal como puede apreciarse en la tabla IV. Así, de las 85 analogías que aparecen en el primer ciclo de la ESO ( $1^{\circ}$ y $2^{\circ}$ de ESO), 46 figuran en los libros de texto de ciencias naturales de $1^{\circ}$ de ESO y 39 en los de $2^{\circ}$ de ESO.

Los textos que muestran el segundo porcentaje más alto de distribución de analogías por materias (Tabla III) son los de física y química de educación secundaria obligatoria $(17,5 \%)$. Los textos de biología y geología de ESO presentan un porcentaje inferior, aunque también alto, de analogías: $14 \%$. Estos datos indican, de nuevo, la preferencia de autores y editores de libros de texto por incorporar las analogías en los textos de niveles más bajos de la educación secundaria para facilitar la comprensión de los conceptos que en ellos figuran. Además, emplean las analogías con mayor frecuencia en los textos de física y química que en los de biología y geología de ESO, ya que los primeros presentan contenidos que resultan ser más complejos, difíciles y abstractos para el aprendizaje de los alumnos que los de biología y geología. Estos últimos son más proclives a describirse con ejemplos del entorno.

Las analogías no se distribuyen por igual en el $2^{\circ}$ ciclo de la $\operatorname{ESO}\left(3^{\circ}\right.$ y $4^{\circ}$ de ESO) entre los textos de física y química. Si se observa la tabla IV se contabilizan 70 analogías de las que 56 figuran en los textos de $3^{\circ}$ de ESO, mientras que 14 figuran en los textos de $4^{\circ}$ de ESO. La razón está en que la mayor parte de los contenidos que figuran en los textos de física y química de $3^{\circ}$ de ESO están relacionados con la química y la electricidad; son contenidos que utilizan conceptos abstractos, de mayor dificultad de comprensión para los alumnos. Los contenidos de los textos de física y química de $4^{\circ}$ de ESO son, mayoritariamente, contenidos de física que -en este nivel de la educación secundaria- presentan un menor grado de abstracción y que pueden explicarse utilizando otros recursos didácticos, como los ejemplos.

La distribución de las analogías entre los textos de biología y geología de $3^{\circ}$ de ESO y de $4^{\circ}$ de ESO es prácticamente homogénea, ya que en la tabla IV se recuentan 56 analogías,
30 de ellas figuran en los textos de $3^{\circ}$ de ESO mientras que las 26 restantes figuran en los textos de $4^{\circ}$ de ESO. Este hecho revela que el grado de abstracción de esta materia en los dos cursos del $2^{\circ}$ ciclo de la ESO es semejante.

La lectura de la tabla III indica que el porcentaje de analogías en los textos de $1^{\circ}$ de bachillerato es también alto: $11,3 \%$ en biología y geología y $10,8 \%$ en física y química. Se infiere que en este curso de $1^{\circ}$ de bachillerato no se mantiene la tendencia definida por los textos de biología y geología y física y química de ESO. Es alto el porcentaje de analogías en los textos de física de $2^{\circ}$ de bachillerato $(10,8 \%)$, mientras que es bajo en los textos de química de $2^{\circ}$ de bachillerato $(7,02 \%)$. Este bajo porcentaje puede estar justificado porque en el cómputo no se ha tenido en cuenta el texto de la editorial Oxford, ya que aún no se había publicado. Con toda probabilidad este texto hubiese permitido aumentar considerablemente el porcentaje en esta materia.

\section{A MODO DE SÍNTESIS}

A partir de un análisis comparativo de las definiciones y posiciones acerca de la idea de analogía se argumenta dicha concepción, vista como todo un proceso de modelización mental, complejo, en el que se conecta, mediante la comparación, el nuevo conocimiento con el que ya tienen los estudiantes. Consustancial a la propuesta se pretende dar coherencia y relación a toda una terminología diferente, usada por distintos autores, como puede ser: análogo, tópico, trama, relación analógica, modelo mental, componentes, atributos, nexos, semejanza estructural, funcional, semántica o superficial. Se explicitan y ejemplifican las características similares que intervienen con este fundamento teórico en la analogía del sistema solar y el átomo de Rutherford.

El análisis de los libros de texto ha permitido conocer la distribución de las analogías en los libros de texto de la muestra de investigación atendiendo a su fecha de publicación, a las etapas educativas (ESO y bachillerato) y materias en cada una de las editoriales, y a los cursos escolares de la educación secundaria.

\section{NOTAS}

${ }^{1}$ Traducidas con la expresión más representativa de la intención del autor, que haga coherente el discurso.

${ }^{2}$ La trama de relaciones que se establece en la analogía se denomina, con bastante frecuencia, relación analógica.

${ }^{3}$ La cantidad promedio se expresa redondeada y con tres cifras significativas.

Tabla IV

Distribución de las analogías por materias y cursos de ESO.

\begin{tabular}{|c|c|c|c|c|c|c|c|}
\hline EDITORIAL & $\begin{array}{c}1^{\circ} \text { ESO } \\
\text { CCNN }\end{array}$ & $\begin{array}{c}2^{\circ} \text { ESO } \\
\text { CCNN }\end{array}$ & $\begin{array}{c}3^{\circ} \text { ESO } \\
\text { F y Q }\end{array}$ & $\begin{array}{c}3^{\circ} \text { ESO } \\
\text { B y G }\end{array}$ & $\begin{array}{c}4^{\circ} \text { ESO } \\
\text { F y Q }\end{array}$ & $\begin{array}{c}4^{\circ} \text { ESO } \\
\text { B y G }\end{array}$ & $\begin{array}{c}\text { TOTAL } \\
\text { ANALOGÍAS }\end{array}$ \\
\hline TOTAL & 46 & 39 & 56 & 30 & 14 & 26 & 211 \\
\hline
\end{tabular}




\section{REFERENCIAS BIBLIOGRÁFICAS}

ARAGÓN, M., BONAT, M., CERVERA, J., MATEOS, J. y OLIVA, J. (1997). Las analogías como estrategia didáctica en la enseñanza de la física y de la química. Enseñanza de las Ciencias, núm. extra, V Congreso, pp. 235-236.

BLOOM, J. (1992). Contextual flexibility: Learning and change from cognitive, sociocultural and physical context perspectives. The history and philosophy of science in science education, 1, pp. 115-125. Ontario: Editorial S. Hills Kingston.

CLEMENT, J. (1993). Using Bridging Analogies and Anchoring Intuitions to Deal with Students'Preconceptions in Physics. Journal of Research in Science Teaching, 30(10), pp. 1241-1257.

DAGHER, Z. (1994). Does the Use of Analogies Contribute to Conceptual Change? Science Education, 78(6), pp. 601-614.

DAGHER, Z. y COSSMAN, G. (1992). Verbal Explanations Given by Science Teachers: Their Nature and Implications. Journal of Research in Science Teaching, 29(4), pp. 361-374.

DAVIS, P. y DAVIDSON, G. (1994). Language is Like the Human Body: Teaching Concepts Through Analogy. Educational Technology, 34(5), pp. 27-32.

DONNELLY, C. y McDANIEL, M. (1993). Use of Analogy in Learning Scientific Concepts. Journal of Experimental Psychology: Learning, Memory and Cognition, 19(4), pp. $975-987$.

DUIT, R. (1991). On the Role of Analogies and Metaphors in Learning Science. Science Education, 75(6), pp. 649-672.

GENTNER, D. (1983). Structure-Mapping: a Theoretical Framework for Analogy. Cognitive Science, 7, pp. 155-170.

GENTNER, D. y FORBUS, K.D. (1996). Analogy, Mental Models, and Conceptual Change. <http://www.grg.ils.nwu.edu/> [Consulta: 01 junio 1998].

GLYNN, S. (1991). Explaining Science Concepts: A Teaching with Analogies Model. The Psychology of Learning Science, pp. 219-240. Londres: Glynn, S., Yeany, R. y Britton (eds.)

GLYNN, S., BRITTON, B., SEMRUD-CLIKEMAN, M. y MUTH, K.D. (1989). Analogical reasoning and problem solving in science textbooks, en J.A. A handbook of creativity: Assessment, theory, and research, pp. 383-398. Nueva York: Glover, R.R. Ronning y C.R. Reynolds (eds.).

GLYNN, S., LAW, M. y DOSTER, E. (1998). Making Text Meaningful: The Role of Analogies. Learning From Text Across Conceptual Domains, pp. 193-208. Nueva Jersey: Cynthia R. Hynd (ed.).

GLYNN, S., RUSSELL, A. y NOAH, D. (1997). Teaching Science Concepts to Children: The Role of Analogies. <http: //www.coe.uga.edu/edpsych/faculty/glynn/twa.html> [Consulta: 23 marzo 1998]

GONZÁLEZ, B.M. (2002). «Las analogías en el proceso en- señanza-aprendizaje de las ciencias de la naturaleza». Tesis doctoral (ISBN: 84-699-9636-3).

GONZÁLEZ, B.M. y MORENO, T. (1998). Las analogías en la enseñanza de las Ciencias. La Docencia de las Ciencias Experimentales en la Enseñanza Secundaria, pp. 204-206. Madrid: II Simposio del Colegio Oficial de Biólogos, Ediciones.

GONZÁLEZ, B.M., MORENO, T. y FERNÁNDEZ, J. (2000). Modelos de enseñanza con analogías. Actas de los XIX Encuentros de Didáctica de las Ciencias Experimentales, pp. 161-169. Madrid: Facultad de Educación de la Universidad Complutense.

HALFORD, G. (1993). Children's Understanding. Nueva Jersey: Lawrence Erlbaum Editors.

HOLYOAK, K. y THAGARD, P. (1989). Analogical Mapping by Constraint Satisfaction. Cognitive Science, 13, pp. 295-355.

IDING, M. (1997). How analogies foster learning from science texts. Instructional Science, 25(4), pp. 233-253.

LAWSON, A. (1993). The Importance of Analogy: A Prelude to the Special Issue. Journal of Research in Science Teaching, 30(10), pp. 1213-1214.

NEWTON, D. y NEWTON, L. (1995). Using Analogy to Help Young Children Understand. Educational Studies, 21(3), pp. 379-393.

OSBORNE, R. y FREYBERG, P. (1985). Learning in science: The implications of children's science. Nueva Zealand: Heinemann Editors.

PIÑAR, I. (1998). Física y química $3^{\circ}$ secundaria. Vizcaya: Oxford University Press España, SA.

ROBERTS, D. (1970). Science as an explanatory mode. Main Currents in Current Thought, 26(5), pp. 131-139.

SIMONS, P. (1984). Instructions with analogies. Journal of Educational Psychology, 76(3), pp. 513-527.

THAGARD, P. (1992). Analogy, Explanation and Education. Journal of Research in Science Teaching, 29(6), pp. 537-544.

THIELE, R. y TREAGUST, D. (1994). The nature and extent of analogies in secondary chemistry textbooks. Instructional Science, 22(1), pp. 61-74.

TREAGUST, D., DUIT, R., JOSLIN, P. y LINDAUER, I. (1992). Science teachers'use of analogies: observations from classroom practice. International Journal of Science Education, 14(4), pp. 413-422.

WONG, E.D. (1993). Understanding the Generative Capacity of Analogies as a Tool for Explanation. Journal of Research in Science Teaching, 30(10), pp. 1259 -1272.

ZOOK, K. y DI VESTA, F. (1991). Instructional Analogies and Conceptual Misrepresentations. Journal of Educational Psychology, 83(2), pp. 246-252. 